MATHEMATICS OF COMPUTATION

Volume 00, Number 0, Pages 000-000

S $0025-5718(\mathrm{XX}) 0000-0$

\title{
OPTIMAL $N$-TERM APPROXIMATION BY LINEAR SPLINES OVER ANISOTROPIC DELAUNAY TRIANGULATIONS
}

\author{
LAURENT DEMARET AND ARMIN ISKE
}

\begin{abstract}
Anisotropic triangulations provide efficient geometrical methods for sparse representations of bivariate functions from discrete data, in particular from image data. In previous work, we have proposed a locally adaptive method for efficient image approximation, called adaptive thinning, which relies on linear splines over anisotropic Delaunay triangulations. In this paper, we prove asymptotically optimal $N$-term approximation rates for linear splines over anisotropic Delaunay triangulations, where our analysis applies to relevant classes of target functions: (a) piecewise linear horizon functions across $\alpha$ Hölder smooth boundaries, (b) functions of $W^{\alpha, p}$ regularity, where $\alpha>2 / p-1$, (c) piecewise regular horizon functions of $W^{\alpha, 2}$ regularity, where $\alpha>1$.
\end{abstract}

\section{INTRODUCTION}

During the last few years, there has been an increasing demand in efficient (i.e., sparse) representations of bivariate functions, especially for images. From the viewpoint of (image) approximation, one is interested in the construction of suitable dictionaries $\mathcal{A}=\left\{\varphi_{j}\right\}_{j \in \mathbb{N}} \subset L^{2}\left([0,1]^{2}\right)$ to obtain asymptotically optimal $N$-term approximations of the form

$$
\left\|f-f_{N}\right\|_{L^{2}\left([0,1]^{2}\right)}^{2}=\mathcal{O}\left(N^{-\alpha}\right) \text { for } N \rightarrow \infty,
$$

where $f_{N}$ is, for any $N \in \mathbb{N}$, a linear combination of $N$ (suitably chosen) elements from $\mathcal{A}$. Moreover, the target $f$ is assumed to lie in a function class $\mathcal{F}_{\alpha} \subset L^{2}\left([0,1]^{2}\right)$ of regular or piecewise regular bivariate functions, whose regularity is reflected by the parameter $\alpha>0$. We say that an asymptotic $N$-term approximation of the form (1.1) has decay rate $\mathcal{O}\left(N^{-\alpha / 2}\right)$ w.r.t. the $L^{2}$-norm.

Tensor product wavelets are classical tools to provide (mildly) nonlinear approximation schemes for image approximation. In this approach, for a given wavelet orthonormal basis of $L^{2}\left([0,1]^{2}\right)$, the $N$-term approximation operator $W_{N}$ associates to any $f \in L^{2}\left([0,1]^{2}\right)$ the $L^{2}$-function $f_{N}=W_{N} f$ obtained by the $N$ largest wavelet coefficients of $f$ (see e.g. [14]). Provided that the chosen wavelet basis satisfies sufficient regularity and decay conditions, the resulting decay rate of the $N$-term approximation in (1.1) is related to the Besov regularity of the target $f \in L^{2}\left([0,1]^{2}\right)$.

More precisely, we have the following equivalence, which directly follows from equation (7.41) in [14], for $p=2, d=2, s=2, \gamma=\alpha, q=\tau$, in combination with [14, Remark 7.6] and the subsequent discussion in Subsection 7.7 of [14] on $N$-term approximations by wavelet decompositions on bounded domains $\Omega \subset \mathbb{R}^{d}$.

2010 Mathematics Subject Classification. Primary. 
Proposition 1.1. For $f \in L^{2}\left([0,1]^{2}\right)$, an asymptotic $N$-term approximation of decay rate $\mathcal{O}\left(N^{-\alpha / 2}\right)$,

$$
\left\|f-W_{N} f\right\|_{L^{2}\left([0,1]^{2}\right)}^{2}=\mathcal{O}\left(N^{-\alpha}\right) \quad \text { for } N \rightarrow \infty,
$$

is achieved if and only if $f \in B_{\tau, \tau}^{\alpha}\left([0,1]^{2}\right)$, where $B_{\tau, \tau}^{\alpha}\left([0,1]^{2}\right)$ is the Besov space of regularity $\alpha$ w.r.t. the $L^{\tau}$-norm, and where $1 / \tau=1 / 2+\alpha / 2$.

However, in cases where $f \in L^{2}\left([0,1]^{2}\right)$ is only piecewise smooth with singularities along (smooth) curves, $N$-term approximations by tensor product wavelets are of the form (cf. [15, eq. (3.5), p. 727])

$$
\left\|f-W_{N} f\right\|_{L^{2}\left([0,1]^{2}\right)}^{2}=\mathcal{O}\left(N^{-1}\right) \quad \text { for } N \rightarrow \infty .
$$

It is well-known that the decay rate $\mathcal{O}\left(N^{-1 / 2}\right)$ in (1.2) is only suboptimal [27].

Different geometrical methods were recently developed to design efficient image approximation schemes, where correlations along curves are essentially taken into account to locally capture the geometry of the given image data. Curvelets $[6,7]$ and shearlets $[21,22]$ are prominent examples for non-adaptive redundant function frames with strong anisotropic directional selectivity.

For piecewise Hölder continuous functions $f$ of second order with discontinuities along $\mathscr{C}^{2}$-curves, Candès and Donoho [7] proved that a best approximation $f_{N}$ to $f$ with $N$ curvelets satisfies the asymptotic decay rate

$$
\left\|f-f_{N}\right\|_{L^{2}\left([0,1]^{2}\right)}^{2}=\mathcal{O}\left(N^{-2}\left(\log _{2} N\right)^{3}\right) \quad \text { for } N \rightarrow \infty .
$$

Up to the $\left(\log _{2} N\right)^{3}$ factor, this curvelet $N$-term approximation rate is asymptotically optimal (see [7, Section 1.3]).

Similar decay rates were proven by Guo and Labate [21] for shearlet frames. We remark that curvelets (and other related approximation schemes) are not adaptive to the assumed regularity of the target $f$. Therefore, the curvelet $N$-term approximation rate in (1.3) does not apply to functions of less regularity, e.g. functions $f$ which are only piecewise $\mathscr{C}^{\alpha}$ with singularities along $\mathscr{C}^{\alpha}$-curves, for $\alpha<2$.

When approximating piecewise regular functions, in particular images, locally adaptive approximation methods are of increasing interest. In fact, during the last few years, several approaches for (locally) adaptive image approximation schemes were developed $[2,10,16,17,23,26,28,29,30,32,33]$, where the approximation schemes are adapted to the (local) image geometry, rather than fixing a basis or a function frame beforehand to approximate $f$.

Recent alternative concepts for locally adaptive approximation methods rely on anisotropic triangulation methods. Due to their simplicity and flexibility, these methods are enjoying an increasing popularity, especially in image approximation. In previous work, we have developed one such image approximation scheme, termed adaptive thinning, which works with linear splines over anisotropic Delaunay triangulations, and which is locally adaptive to the geometric regularity of the image. As demonstrated in $[11,13]$, adaptive thinning leads to an efficient and competitive image compression method at computational complexity $\mathcal{O}(N \log (N))$. Related methods for image approximations by anisotropic triangulations are in [5, 8, 9, 25], see the survey [12] for a comparison of these image approximation methods.

Yet it remains to prove asymptotic error bounds of the form (1.1) for image approximation methods relying on linear splines over anisotropic triangulations. 
In this paper, we show that linear splines over locally adaptive anisotropic Delaunay triangulations lead to asymptotically optimal $N$-term approximation rates

$$
\left\|f-f_{N}\right\|_{L^{2}\left([0,1]^{2}\right)}^{2} \leq C N^{-\alpha} \quad \text { for } \alpha \in(1,2]
$$

for relevant classes of target functions $f$, including

- piecewise linear horizon functions across $\alpha$ Hölder smooth boundaries,

- functions of $W^{\alpha, p}$ regularity, where $\alpha>2 / p-1$,

- piecewise regular horizon functions of $W^{\alpha, 2}$ regularity, where $\alpha>1$.

Our constructive approach taken for the computation of the anisotropic Delaunay triangulations essentially depends on the local regularity of the target function $f$. The resulting approximation method applies in particular to the relevant case of piecewise Hölder continuous horizon functions $f$ of order $\alpha \in(1,2]$ with singularities along $\alpha$ Hölder smooth boundaries.

The outline of this paper is as follows. In the following Section 2, we briefly introduce linear splines over Delaunay triangulations, where we recall some of their basic properties. In Section 3, we show that linear splines over locally adaptive Delaunay triangulations lead to asymptotic $N$-term estimates of the form (1.4) for piecewise linear-affine horizon functions with $\alpha$ Hölder smooth boundary. Then, in Section 4 we first discuss a classical result by Birman-Solomjak [4], which states that piecewise affine-linear functions over quadtree partitions provide optimal approximation rates of the form (1.4) for regular functions $f \in W^{\alpha, p}\left([0,1]^{2}\right)$, where $\alpha>2 / p-1$. We transfer the Birman-Solomjak result to the special case of linear spline approximation over locally adaptive Delaunay triangulations. Finally, in Section 5, we combine the results from Sections 3-4 to obtain $N$-term approximations of the form (1.4) for piecewise regular horizon functions across Hölder smooth horizon boundaries.

\section{Linear Splines over Delaunay Triangulations}

In this section, we introduce the basic ingredients which are required for the subsequent analysis in the following Sections 3-5. But let us make a few preliminary remarks concerning their approximation behaviour.

When using triangulation methods for image approximation to piecewise smooth images $f$, one should consider anisotropic triangular meshes, whose triangular elements are well-adapted to the local image geometry. Therefore, we are interested in the construction of locally adaptive triangulations, whose long and thin triangles are aligned with the curve singularities of $f$ to improve the accuracy of the resulting approximation scheme. On the other hand, in regions of higher regularity, larger and isotropic triangles should be used in order to increase the efficiency of the resulting approximation scheme.

We can combine our basic requirements, especially for local adaptivity and for computational efficiency, by using Delaunay triangulations. Delaunay triangulations are well-known for providing stable and flexible approximation schemes, where their triangular meshes are efficient to compute and to maintain. Moreover, due to their uniqueness, Delaunay triangulations entirely avoid the coding costs for the connectivities between their nodes.

Therefore, we prefer to work with linear splines over locally adaptive Delaunay triangulations, whose basic properties are explained as follows. 
2.1. Conformal and Delaunay Triangulations. Let us start our discussion by introducing conformal triangulations.

Definition 2.1. A conformal triangulation $\mathcal{T} \equiv \mathcal{T}(Y)$ of a discrete planar point set $Y$ is a set $\mathcal{T}=\{T\}_{T \in \mathcal{T}}$ of triangles satisfying the following properties.

(a) the vertex set of $\mathcal{T}$ is $Y$;

(b) any pair of two distinct triangles in $\mathcal{T}$ intersect at most at one common vertex or along one common edge;

(c) the convex hull $\operatorname{conv}(Y)$ of $Y$ coincides with the area covered by the union of the triangles in $\mathcal{T}$.

In the following discussion, we require $\operatorname{conv}(Y)=[0,1]^{2}$, so that $\mathcal{T}$ is a partitioning of the (image) domain $[0,1]^{2}$. Now let us turn to Delaunay triangulations.

Definition 2.2. The Delaunay triangulation $\mathcal{D}(Y)$ of a discrete planar point set $Y$ is a conformal triangulation of $Y$, where no circumcircle of a triangle $T \in \mathcal{D}(Y)$ contains any point from $Y$ in its interior.

We recall three important properties of Delaunay triangulations.

(a) The Delaunay triangulation $\mathcal{D}$ of $Y$ is unique, provided that no four points in $Y$ are co-circular. We remark that there are efficient computational methods for choosing, on given $Y$, a Delaunay triangulation of $Y$ without ambiguity (see [19]). Therefore, we will from now assume that $\mathcal{D}$ is the unique Delaunay triangulation of $Y$.

(b) On a given point set $Y$ of size $N=|Y|$, its (unique) Delaunay triangulation $\mathcal{D}$ can be computed in $\mathcal{O}(N \log (N))$ steps.

(c) For any Delaunay triangulation $\mathcal{D}$ of a point set $Y$, its dual graph is the Voronoi diagram $\mathcal{V}$ of $Y$.

To further explain property (c), let us first introduce Voronoi diagrams.

Definition 2.3. For any finite planar point set $Y$, the Voronoi diagram $\mathcal{V}(Y)$ of $Y$ is a planar graph consisting of the Voronoi tiles

$$
V_{Y}(y)=\left\{x \in \mathbb{R}^{2}:\|x-y\|_{2}=\min _{z \in Y}\|x-z\|_{2}\right\} \quad \text { for } y \in Y,
$$

each containing, for $y \in Y$, all points in the plane whose nearest point in $Y$ is $y$.

Now, the Delaunay triangulation of $Y$ is dual to the Voronoi diagram in the following way. Any pair $\left(y_{1}, y_{2}\right)$ of two distinct points $y_{1}, y_{2} \in Y$ are said to be a pair of Voronoi neighbours, iff the intersection $V_{Y}\left(y_{1}\right) \cap V_{Y}\left(y_{2}\right)$ of their Voronoi tiles is a non-degenerate edge in $\mathcal{V}_{Y}$. Now, for any pair of Voronoi neighbours $\left(y_{1}, y_{2}\right)$, the straight line $\left[y_{1}, y_{2}\right]$ between $y_{1}$ and $y_{2}$ is an edge in the Delaunay triangulation $\mathcal{D}(Y)$ of $Y$. In fact, by connecting all possible Voronoi neighbours in $\mathcal{V}(Y)$, we obtain a planar graph yielding the Delaunay triangulation $\mathcal{D}(Y)$ of $Y$. For further details concerning the duality between Delaunay triangulations and Voronoi diagrams we refer to the textbook [31].

2.2. Linear Splines over Conformal Triangulations. We associate with any conformal triangulation $\mathcal{T}$ the finite dimensional linear function space

$$
\mathcal{S}_{\mathcal{T}}:=\left\{g \in \mathscr{C}\left([0,1]^{2}\right):\left.g\right|_{T} \in \mathcal{P}_{1} \text { for all } T \in \mathcal{T}\right\}
$$

of all linear splines over $\mathcal{T}$, containing all globally continuous functions over $[0,1]^{2}$ whose restriction to any triangle $T \in \mathcal{T}$ is a linear polynomial. Therefore, $\mathcal{P}_{1}$ in the 
above definition of $\mathcal{S}_{\mathcal{T}}$ denotes the linear space of all bivariate linear polynomials. Recall that the dimension of $\mathcal{S}_{\mathcal{T}}$ is the number $|Y|$ of vertices $Y$.

Note that for any function $f \in \mathscr{C}\left([0,1]^{2}\right)$, there is a unique linear spline interpolant $s \in \mathcal{S}_{\mathcal{T}}$ to $f$ over the vertices $Y$ of $\mathcal{T}$ satisfying $\left.s\right|_{Y}=\left.f\right|_{Y}$. In particular, any linear spline $s \in \mathcal{S}_{\mathcal{T}}$ is uniquely determined by its values at the vertices $Y$ of $\mathcal{T}$.

In the following Sections 3-5 we prove asymptotically optimal $N$-term approximation rates of the form (1.4) for linear spline interpolation over (conformal) Delaunay triangulations. To this end, we construct sequences of (conformal) Delaunay triangulations $\left\{\mathcal{D}_{N}\right\}_{N \in \mathbb{N}}$, such that there are constants $C, M>0$ (independent of $N$ ) satisfying the following two properties.

(a) The number $\left|Y_{N}\right|$ of vertices in $\mathcal{D}_{N}$ is bounded above by $\left|Y_{N}\right| \leq M \times N$;

(b) the $L^{2}$-approximation error can be bounded above by

$$
\left\|f-f_{N}\right\|_{L^{2}\left([0,1]^{2}\right)}^{2} \leq C N^{-\alpha},
$$

where $f_{N} \in \mathcal{S}\left(\mathcal{D}_{N}\right)$ is the unique linear interpolant to $f$ at $Y_{N}$, and $\alpha>0$ is essentially related to the regularity of $f$, as detailed in Sections 3-5.

\section{Approximation of Horizon Functions}

Horizon functions [17] are popular and simple prototypes for piecewise smooth images with discontinuities along Hölder smooth curves. In this section, we prove asymptotically optimal $N$-term approximations of horizon functions by linear splines over conformal triangulations.

To introduce the class of horizon functions, first recall that for any $\alpha=r+\beta$, with $r \in \mathbb{N}_{0}$ and $\beta \in(0,1]$,

$$
\mathscr{C}^{\alpha}[0,1]:=\left\{g \in \mathscr{C}^{r}[0,1]:\left|g^{(r)}(x)-g^{(r)}(y)\right| \leq C|x-y|^{\beta} \text { for all } x, y \in[0,1]\right\}
$$

is the linear space of $\alpha$-Hölder smooth functions over $[0,1]$. Moreover, $\mathscr{C}^{\alpha}[0,1]$ is by

$$
|g|_{\mathscr{C}^{\alpha}([0,1])}:=\inf \left\{C:\left|g^{(r)}(x)-g^{(r)}(y)\right| \leq C|x-y|^{\beta} \text { for all } x, y \in[0,1]\right\}
$$

equipped with the usual semi-norm.

In our following analysis, we require $\alpha \in(1,2]$, i.e., we assume $\alpha=1+\beta$ for $\beta=\alpha-1 \in(0,1]$, in which case for any $\alpha$-Hölder smooth function $g \in \mathscr{C}^{\alpha}([0,1])$,

$$
|g|_{\mathscr{C}^{\alpha}([0,1])}=\inf \left\{C:\left|g^{\prime}(x)-g^{\prime}(y)\right| \leq C|x-y|^{\alpha-1} \text { for all } x, y \in[0,1]\right\}
$$

is the semi-norm of $g$ in $\mathscr{C}^{\alpha}([0,1])$. Note that $g^{\prime} \in \mathscr{C}^{\alpha-1}([0,1])$, where

$$
\left|g^{\prime}\right|_{\mathscr{C}^{\alpha-1}([0,1])}=|g|_{\mathscr{C}^{\alpha}([0,1])} \quad \text { for all } g \in \mathscr{C}^{\alpha}([0,1]) .
$$

In the remainder of this paper, we let

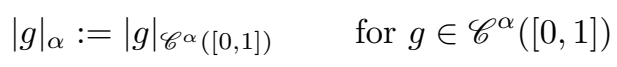

for notational convenience.

Now the class of $\alpha$-horizon functions comprises all piecewise affine-linear functions with one discontinuity along an $\alpha$-Hölder smooth horizon boundary. To be more precise, we give the following definition. 
Definition 3.1. For any $\alpha \in(1,2]$, a function $f:[0,1]^{2} \rightarrow \mathbb{R}$ is said to be an $\alpha$-horizon function, iff it has the form

$$
f(x, y):= \begin{cases}p(x, y) & \text { for } y \leq g(x), \\ q(x, y) & \text { otherwise, }\end{cases}
$$

for some affine-linear functions $p, q: \mathbb{R}^{2} \rightarrow \mathbb{R}$ and with $g \in \mathscr{C}^{\alpha}([0,1])$ satisfying $g([0,1]) \subset(0,1)$. The $\alpha$-Hölder smooth function $g \in \mathscr{C}^{\alpha}([0,1])$ is called the horizon boundary of $f$.

We remark that our definition for $\alpha$-horizon functions differs from that in [17], where piecewise constant horizons (rather than piecewise affine-linear horizons) are used. This slight extension of the definition in [17] leads us to a slightly larger target space (of piecewise affine-linear horizons), which better complies with the concept of our approximation scheme, where we use linear splines, i.e., piecewise affine-linear functions over conformal triangulations. But otherwise our extension in Definition 3.1 to that in [17] is immaterial for the subsequent analysis.

3.1. Optimal Decay Rates on Conformal Triangulations. Now let us turn to the approximation of $\alpha$-horizon functions by linear splines over anisotropic Delaunay triangulations. Our first result relies on a rather straightforward application of the classical univariate spline theory. In fact, we can immediately extend wellknown results from univariate spline approximation to bivariate approximation of horizon functions as follows. We remark that a similar result for $\mathscr{C}^{2}$-curves is given in [27, pp. 404-405].

Proposition 3.2. For $\alpha \in(1,2]$, let $f$ be an $\alpha$-horizon function. Then, there exist constants $C, M>0$ (independent of $N$ ), such that for any $N \in \mathbb{N}$ there is a conformal triangulation $\mathcal{T}_{N}$ with $\left|\mathcal{T}_{N}\right| \leq M \times N$ vertices satisfying

$$
\left\|f-f_{N}\right\|_{L^{2}[0,1]^{2}}^{2} \leq C N^{-\alpha},
$$

where $f_{N} \in \mathcal{S}_{\mathcal{T}_{N}}$ is the spline interpolant to $f$ at the vertices of $\mathcal{T}_{N}$.

Proof. We split the proof into three steps.

Step 1. In the first step, we apply univariate spline interpolation to approximate the horizon boundary $g \in \mathscr{C}^{\alpha}[0,1]$. To this end, let $S_{N}(g)$ be the unique linear spline interpolant to $g$ at uniform knots, $x_{i}=i / N, i=0, \ldots, N$, of mesh width $h=1 / N$.

In this case, the approximation error between $g$ and $S_{N}(g)$ can, over any interval $\left[x_{i}, x_{i+1}\right]$, be represented as

$$
\left|g(x)-\left(S_{N} g\right)(x)\right|=\left|g(x)-g\left(x_{i}\right)-\frac{g\left(x_{i+1}\right)-g\left(x_{i}\right)}{x_{i+1}-x_{i}}\left(x-x_{i}\right)\right| .
$$

Since $g^{\prime} \in \mathscr{C}^{\alpha-1}([0,1])$, we have

$$
\left|\frac{g\left(x_{i+1}\right)-g\left(x_{i}\right)}{x_{i+1}-x_{i}}-g^{\prime}\left(x_{i}\right)\right| \leq C\left|x_{i+1}-x_{i}\right|^{\alpha-1}=C h^{\alpha-1},
$$

for some constant $C>0$, which in turn implies

$$
\left|g(x)-\left(S_{N} g\right)(x)\right|=\left|g(x)-g\left(x_{i}\right)-\left(g^{\prime}\left(x_{i}\right)+\mathcal{O}\left(h^{\alpha-1}\right)\right)\left(x-x_{i}\right)\right| .
$$

On the other hand, by Taylor series expansion, we have

$$
g(x)=g\left(x_{i}\right)+g^{\prime}\left(x_{i}\right)\left(x-x_{i}\right)+\mathcal{O}\left(\left|x-x_{i}\right|^{\alpha}\right)
$$


so that (3.2) can further be rewritten as

$$
\left|g(x)-\left(S_{N} g\right)(x)\right|=\mathcal{O}\left(\left|x-x_{i}\right|^{\alpha}\right)+\mathcal{O}\left(h^{\alpha}\right)=\mathcal{O}\left(h^{\alpha}\right) .
$$

This yields the uniform bound

$$
\left\|g-S_{N}(g)\right\|_{L^{\infty}([0,1])} \leq C h^{\alpha}=C N^{-\alpha}
$$

for some constant $C>0$ independent of $N$.

Step 2. We wish to approximate $f$ by functions $f_{N}:[0,1]^{2} \rightarrow \mathbb{R}$ of the form

$$
f_{N}(x, y):= \begin{cases}p(x, y) & \text { for } y \leq\left(S_{N} g\right)(x)-\varepsilon_{N}, \\ q(x, y) & \text { for } y \geq\left(S_{N} g\right)(x)+\varepsilon_{N}, \\ g_{N}(x, y) & \text { otherwise }\end{cases}
$$

for a sequence of sufficiently small constants $\varepsilon_{N}>0$ (to be specified in step 3), and where the functions $g_{N}:[0,1]^{2} \rightarrow \mathbb{R}$ are required to be uniformly bounded on $[0,1]^{2}$ by $\left\|g_{N}\right\|_{L^{\infty}\left([0,1]^{2}\right)} \leq\|f\|_{L^{\infty}\left([0,1]^{2}\right)}$, so that, in particular, the functions $f_{N}:[0,1]^{2} \rightarrow \mathbb{R}$ are in this case also uniformly bounded on $[0,1]^{2}$, i.e., we have

$$
\left\|f_{N}\right\|_{L^{\infty}\left([0,1]^{2}\right)} \leq\|f\|_{L^{\infty}\left([0,1]^{2}\right)} \quad \text { for all } N \in \mathbb{N} .
$$

Moreover, note that $f_{N}$ coincides with $f$ outside the $\varepsilon_{N}$-corridor

$$
K_{\varepsilon_{N}}:=\left\{(x, y) \in[0,1]^{2}:\left|y-\left(S_{N} g\right)(x)\right| \leq \varepsilon_{N}\right\},
$$

so that

$$
\begin{aligned}
\left\|f-f_{N}\right\|_{L^{2}\left([0,1]^{2}\right)}^{2} & =\int_{K_{\varepsilon_{N}}}\left|f(x, y)-f_{N}(x, y)\right|^{2} d x d y \\
& \leq 4\|f\|_{L^{\infty}\left([0,1]^{2}\right)}^{2} \int_{K_{\varepsilon_{N}}} d x d y=8\|f\|_{L^{\infty}\left([0,1]^{2}\right)^{2}}^{2} .
\end{aligned}
$$

Step 3. Finally, we construct a conformal triangulation $\mathcal{T}_{N}$, whose associated linear spline interpolant to $f$ is a function of the form $f_{N}$ in (3.3). To this end, we regard the following three sets of (strictly) convex quadrilaterals.

The first set, $\mathcal{Q}^{+}$, contains all quadrilaterals with vertices

$$
\left(x_{i},\left(S_{N} g\right)\left(x_{i}\right)+\varepsilon_{N}\right),\left(x_{i+1},\left(S_{N} g\right)\left(x_{i+1}\right)+\varepsilon_{N}\right),\left(x_{i}, 1\right),\left(x_{i+1}, 1\right),
$$

the second set, $\mathcal{Q}^{-}$, contains all quadrilaterals with vertices

$$
\left(x_{i},\left(S_{N} g\right)\left(x_{i}\right)-\varepsilon_{N}\right),\left(x_{i+1},\left(S_{N} g\right)\left(x_{i+1}\right)-\varepsilon_{N}\right),\left(x_{i}, 0\right),\left(x_{i+1}, 0\right),
$$

for $i=0, \ldots, N-1$, and the third set, $\mathcal{Q}_{\varepsilon_{N}}$, contains all quadrilaterals with vertices

$$
\left(x_{i},\left(S_{N} g\right)\left(x_{i}\right) \pm \varepsilon_{N}\right),\left(x_{i+1},\left(S_{N} g\right)\left(x_{i+1}\right) \pm \varepsilon_{N}\right) \quad \text { for } i=0, \ldots, N-1 .
$$

Now, we triangulate each quadrilateral $Q \in \mathcal{Q}^{ \pm} \cup \mathcal{Q}_{\varepsilon_{N}}$ by splitting $Q$ across one of its two diagonals. This then yields a conformal triangulation $\mathcal{T}_{N}$ of $[0,1]^{2}$, whose vertices are given by the $4(N+1)$ vertices of the quadrilaterals in $\mathcal{Q}^{ \pm} \cup \mathcal{Q}_{\varepsilon_{N}}$, see Figure 1 (b) for illustration.

Moreover, the unique linear spline interpolant $f_{N} \in \mathcal{S}_{\mathcal{T}_{N}}$ to $f$ satisfying $f_{N} \equiv f$ on $\mathcal{Q}^{ \pm}$has the desired form (3.3), so that

$$
\left\|f-f_{N}\right\|_{L^{2}\left([0,1]^{2}\right)}^{2} \leq 8\|f\|_{L^{\infty}\left([0,1]^{2}\right)}^{2} \varepsilon_{N} .
$$




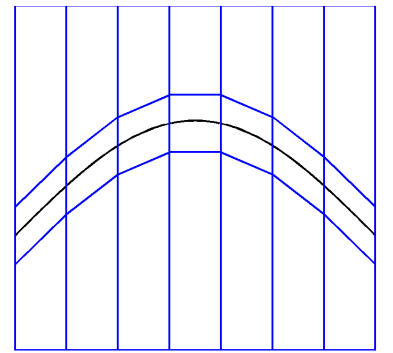

(a)

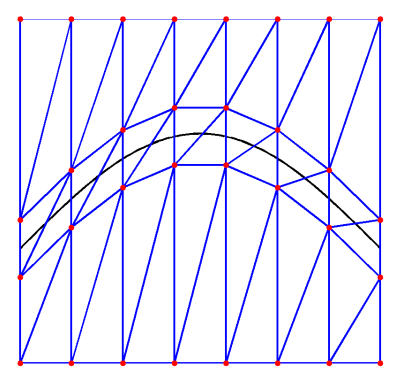

(b)

Figure 1. (a) Approximation of the horizon boundary $g$ by linear spline $S_{N}(g)$, (b) conformal triangulation of vertices $\mathcal{Q}^{ \pm}$and $\mathcal{Q}_{\epsilon_{N}}$.

Now we finally let $\varepsilon_{N}:=h^{\alpha}=N^{-\alpha}$, which then yields the stated error estimate in (3.1) for $C=8\|f\|_{L^{\infty}\left([0,1]^{2}\right)}^{2}$. This completes our proof.

Remark 3.3. The decay rate $\mathcal{O}\left(N^{-\alpha / 2}\right)$ in (3.1) is optimal, i.e., no polynomial depth search dictionary can achieve better decay rates for the class of $\alpha$-horizon functions. This result, in fact, is proven in [24] by using arguments from estimation theory.

3.2. Optimal Decay Rates on Delaunay Triangulations. Note that the triangulations $\mathcal{T}_{N}$ constructed in Proposition 3.2 are anisotropic. In fact, smaller triangles are aligned with the horizon boundary $g$, whereas larger triangles are in smooth areas of $f$. The triangulations $\mathcal{T}_{N}$ are conformal but not necessarily Delaunay triangulations of the vertices $\mathcal{Q}^{ \pm} \cup \mathcal{Q}_{\varepsilon_{N}}$.

In this section, we construct a sequence of Delaunay triangulations $\mathcal{D}_{N}$ of size $\left|\mathcal{D}_{N}\right| \leq M \times N$, with some $M>0$ independent of $N$, such that the associated sequence of linear spline interpolants $f_{N} \in \mathcal{S}_{\mathcal{D}_{N}}$ to $f$ satisfies the error bound

$$
\left\|f-f_{N}\right\|_{L^{2}\left([0,1]^{2}\right)}^{2} \leq C N^{-\alpha}
$$

for some $C>0$ independent of $N$. The Delaunay triangulations $\mathcal{D}_{N}$ are obtained by triangulating the individual quadrilaterals in $\mathcal{Q}^{ \pm}$and $\mathcal{Q}_{\varepsilon_{N}}$ (from the proof of Proposition 3.2) according to the Delaunay criterion. To see this, we first prove the following lemma, which constructs a Delaunay triangulation preserving the upper and the lower boundary line of the corridor $K_{\varepsilon_{N}}$.

Lemma 3.4. For $\alpha \in(1,2]$ let $g \in \mathscr{C}^{\alpha}([0,1])$ with $g([0,1]) \subset(0,1)$. Moreover, let

$$
Y_{n}=\bigcup_{i=0}^{n}\left\{p_{i, n}^{+}, p_{i, n}^{-}\right\} \cup\{(0,0),(0,1),(1,0),(1,1)\},
$$

where

$$
p_{i, n}^{ \pm}=\left(x_{i, n}, g\left(x_{i, n}\right) \pm \varepsilon_{n}\right) \quad \text { for } i=0, \ldots, n,
$$

for $\varepsilon_{n}=|g|_{\alpha} / n^{\alpha}$. Then there is a non-negative integer $N \in \mathbb{N}$, such that for all $n \geq N$ the Delaunay triangulation $\mathcal{D}\left(Y_{n}\right)$ of $Y_{n}$ contains all (horizontal) edges

$$
\left[p_{i, n}^{+}, p_{i+1, n}^{+}\right] \quad \text { and } \quad\left[p_{i, n}^{-}, p_{i+1, n}^{-}\right] \quad \text { for } i=0, \ldots, n-1
$$

and all (vertical) edges $\left[p_{i, n}^{-}, p_{i, n}^{+}\right]$for $i=0, \ldots, n$. 
Proof. Note that by $g([0,1]) \subset(0,1)$ we have $Y_{n} \subset[0,1]^{2}$ for $n$ large enough.

Let us first make some notational preparations. In the remainder of this proof it is convenient to let $g_{i, n}=g\left(x_{i, n}\right)$ for $i=0, \ldots, n$. Moreover, we let

$$
s_{i, n}=\frac{g_{i+1, n}-g_{i, n}}{x_{i+1, n}-x_{i, n}}=n\left(g_{i+1, n}-g_{i, n}\right) \quad \text { for } i=0, \ldots, n-1
$$

denote the slope of the linear spline interpolant $S_{n}(g)$ to $g$ on the interval $\left[x_{i, n}, x_{i+1, n}\right]$. Note that, since $g \in \mathscr{C}^{\alpha}([0,1])$, we have the uniform bound

$$
\left|s_{i, n}\right| \leq|g|_{\alpha} \quad \text { for all } 1 \leq i \leq n \text { and } n \in \mathbb{N} .
$$

Let $C_{i, n}$ be the circumcircle of the triangle $T_{i, n}$ with vertices $p_{i, n}^{-}, p_{i, n}^{+}$and $p_{i+1, n}^{-}$, and let $c_{i, n}$ denote the centre of $C_{i, n}$. Note that $c_{i, n}$ is given by the intersection between the perpendicular bisections of the two segments $\left[p_{i, n}^{-}, p_{i, n}^{+}\right]$and $\left[p_{i, n}^{-}, p_{i+1, n}^{-}\right]$, see Figure 2 for illustration.

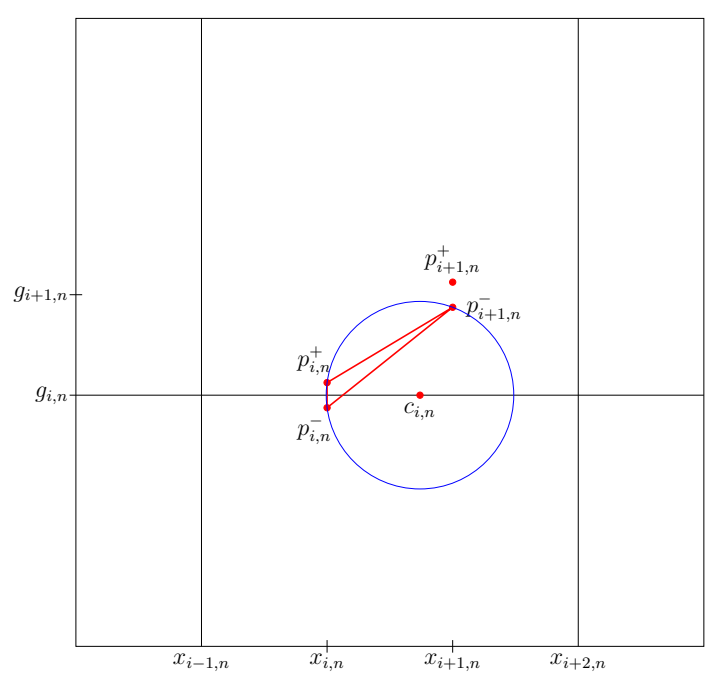

Figure 2. Circumcircle $C_{i, n}$ of triangle $T_{i, n}$ with vertices $p_{i, n}^{ \pm}$and $p_{i+1, n}^{-}$. Note that the centre $c_{i, n}$ of $C_{i, n}$ is given by the intersection between the two perpendicular bisections of the line segments $\left[p_{i, n}^{-}, p_{i, n}^{+}\right]$and $\left[p_{i, n}^{-}, p_{i+1, n}^{-}\right]$.

Now let us determine the two coordinates of $c_{i, n}=\left(c_{x}, c_{y}\right)$. First recall that

$$
p_{i, n}^{ \pm}=\left(x_{i, n}, g_{i, n} \pm \varepsilon_{n}\right) \quad \text { for } i=0, \ldots, n,
$$

which immediately yields $c_{y}=g_{i, n}$. As for the computation of $c_{x}$, let $p_{i+1 / 2, n}^{-}$be the midpoint of the line segment $\left[p_{i, n}^{-}, p_{i+1, n}^{-}\right]$, whose coordinates are given by

$$
p_{i+1 / 2, n}^{-}=\left(x_{i, n}+\frac{1}{2 n}, g_{i, n}-\varepsilon_{n}+\frac{s_{i, n}}{2 n}\right) .
$$

The orthogonality of the line segments $\left[p_{i, n}^{-}, p_{i+1, n}^{-}\right]$and $\left[c_{i, n}, p_{i+1 / 2, n}^{-}\right]$,

$$
0=\left(p_{i+1, n}^{-}-p_{i, n}^{-}\right)^{T}\left(c_{i, n}-p_{i+1 / 2, n}^{-}\right)=\left(\frac{1}{n}, \frac{s_{i, n}}{n}\right)^{T}\left(c_{x}-x_{i, n}-\frac{1}{2 n}, \varepsilon_{n}-\frac{s_{i, n}}{2 n}\right),
$$


yields

$$
c_{x}=x_{i, n}+\frac{1}{2 n}+s_{i, n}\left(\frac{s_{i, n}}{2 n}-\varepsilon_{n}\right)=x_{i, n}+\frac{1}{n}\left(\frac{s_{i, n}^{2}+1}{2}-\frac{s_{i, n}|g|_{\alpha}}{n^{\alpha-1}}\right)
$$

for the other coordinate of the centre $c_{i, n}=\left(c_{x}, c_{y}\right)$.

Next, let us compute the radius $r_{i, n}$ of $C_{i, n}$, as given by the distance between $c_{i, n}$ and $p_{i, n}^{-}=\left(x_{i, n}, g_{i, n}-\varepsilon_{n}\right)$, and so we obtain

$$
\begin{aligned}
r_{i, n}^{2} & =\varepsilon_{n}^{2}+\frac{1}{n^{2}}\left(\frac{s_{i, n}^{2}+1}{2}-\frac{s_{i, n}|g|_{\alpha}}{n^{\alpha-1}}\right)^{2} \\
& =\frac{1}{n^{2}}\left[\frac{|g|_{\alpha}^{2}}{n^{2(\alpha-1)}}+\left(\frac{s_{i, n}^{2}+1}{2}-\frac{s_{i, n}|g|_{\alpha}}{n^{\alpha-1}}\right)^{2}\right] .
\end{aligned}
$$

Due to the uniform bound on $s_{i, n}$ in (3.5), we can conclude $r_{i, n}=\mathcal{O}(1 / n)$, for $n \rightarrow \infty$, i.e., we have

$$
r_{i, n} \leq \frac{C_{r}}{n} \quad \text { for all } n \in \mathbb{N}
$$

for some constant $C_{r}>0$ that does not depend on $n$.

We split the remainder of the proof into the following special cases, where we tacitly assume from now and throughout this proof that $n$ is large enough.

Case 1. Suppose that $s_{i, n}>0$ for the slope of $S_{n}(g)$ on $\left[x_{i, n}, x_{i+1, n}\right]$.

Due to the representation of $c_{x}$ in (3.6) and the uniform bound on $s_{i, n}$ in (3.5), we see that $x_{i, n}<c_{x}$. Moreover, from the decay of its radius $r_{i, n}$ in (3.8), we can conclude that the circle $C_{i, n}$ is contained in the strip $\left(x_{i-1, n}, 1\right] \times[0,1]$. But this implies that all points $\left\{p_{k, n}^{ \pm}: k<i\right\}$ lie outside the circle $C_{i, n}$.

Recall that the three vertices $p_{i, n}^{ \pm}, p_{i+1, n}^{-}$of $T_{i, n}$ lie on the boundary of $C_{i, n}$.

Now let us check the point $p_{i+1, n}^{+}$. To this end, we compare the distances between the centre $c_{i, n}$ and the points $p_{i+1, n}^{ \pm}=\left(x_{i+1, n}, g_{i+1, n} \pm \varepsilon_{n}\right)$, given by

$$
\left\|p_{i+1, n}^{ \pm}-c_{i, n}\right\|^{2}=\left(x_{i+1, n}-c_{x}\right)^{2}+\left(g_{i+1, n}-g_{i, n} \pm \varepsilon_{n}\right)^{2} .
$$

Since $g_{i+1, n}-g_{i, n}>0$, we can conclude that the distance between $p_{i+1, n}^{+}$and $c_{i, n}$ is larger than the distance between $p_{i+1, n}^{-}$and $c_{i, n}$. Therefore, also the point $p_{i+1, n}^{+}$ lies outside the circle $C_{i, n}$.

Case 1a. Suppose $0<s_{i, n}<1$. Given the representation of $c_{x}$ in (3.6), we see that $c_{x}-x_{i, n}<1 / n$, and so $x_{i, n}<c_{x}<x_{i+1, n}$. Likewise, by the representation of $r_{i, n}$ in (3.7), we obtain $r_{i, n}<1 / n$. Therefore, we can conclude that the circle $C_{i, n}$ is contained in the strip $\left(x_{i-1, n}, x_{i+2, n}\right) \times[0,1]$. But this already implies that none of the points $\left\{p_{i, n}^{ \pm}: 0 \leq i \leq n\right\}$ lies in the interior of the circle $C_{i, n}$. Only the three vertices $p_{i, n}^{ \pm}, p_{i+1, n}^{-}$of triangle $T_{i, n}$ lie on the boundary of $C_{i, n}$.

Case $1 b$. Suppose $s_{i, n} \geq 1$.

We first show that no point from $\left\{p_{i+k, n}^{-}: k=2, \ldots, n-i\right\}$ lies in the interior of the circle $C_{i, n}$. Since $g^{\prime} \in \mathscr{C}^{\alpha-1}([0,1])$ and by the mean value theorem, there are 
intermediate points $\xi_{1} \in\left(x_{i, n}, x_{i+1, n}\right)$ and $\xi_{2} \in\left(x_{i+1, n}, x_{i+2, n}\right)$ satisfying

$$
\begin{aligned}
\left|s_{i+1, n}-s_{i, n}\right| & =\left|\frac{g_{i+2, n}-g_{i+1, n}}{x_{i+2, n}-x_{i+1, n}}-\frac{g_{i+1, n}-g_{i, n}}{x_{i+1, n}-x_{i, n}}\right| \\
& =\left|g^{\prime}\left(\xi_{2}\right)-g^{\prime}\left(\xi_{1}\right)\right| \leq\left|g^{\prime}\right|_{\alpha-1} \cdot\left|\xi_{2}-\xi_{1}\right|^{\alpha-1} \leq \frac{C_{g}}{n^{\alpha-1}},
\end{aligned}
$$

for all $i=0, \ldots, n-2$, where we let $C_{g}=2^{\alpha-1} \cdot\left|g^{\prime}\right|_{\alpha-1}$. But this implies

$$
s_{i, n}-s_{i+k, n} \leq\left|s_{i+k, n}-s_{i, n}\right| \leq \sum_{j=0}^{k-1}\left|s_{i+j+1, n}-s_{i+j, n}\right| \leq k \frac{C_{g}}{n^{\alpha-1}} .
$$

Now let $t_{i, n}$ denote the slope of the tangent to the circle $C_{i, n}$ at the point $p_{i+1, n}^{-}$. Note that $t_{i, n}=-1 / t_{i, n}^{\perp}$, where $t_{i, n}^{\perp}$ is the slope of the straight line passing through the centre $c_{i, n}$ and $p_{i+1, n}^{-}$, see Figure 3 .

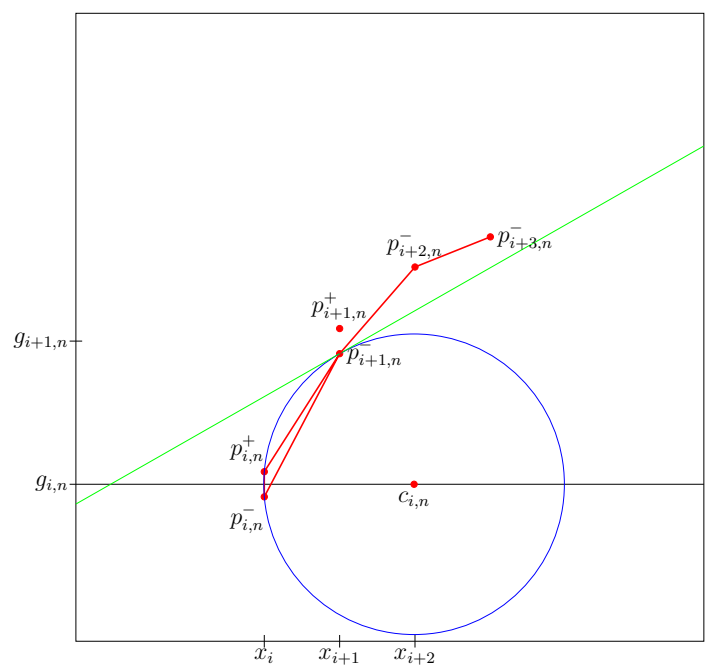

Figure 3. Case 1b. $s_{i, n} \geq 1$. The points $p_{i+k, n}^{ \pm}, k=1, \ldots, n-i$, and the interior of circle $C_{i, n}$ are separated by the tangent of $C_{i, n}$ at $p_{i+1, n}^{-}$. Note that for $s_{i, n}>0$ the ordinate of $p_{i+1, n}^{-}$is necessarily larger than the ordinate of $p_{i, n}^{-}$.

Recalling the coordinates of $c_{i, n}=\left(c_{x}, c_{y}\right)$ and $p_{i+1, n}^{-}=\left(x_{i+1, n}, g_{i+1, n}-\varepsilon_{n}\right)$, we get

$$
t_{i, n}=\frac{x_{i, n}+\frac{1}{n}\left(\frac{s_{i, n}^{2}+1}{2}-\frac{s_{i, n}|g|_{\alpha}}{n^{\alpha-1}}\right)-x_{i+1, n}}{\frac{s_{i, n}}{n}-\varepsilon_{n}}=\frac{\frac{s_{i, n}^{2}-1}{2}-\frac{s_{i, n}|g|_{\alpha}}{n^{\alpha-1}}}{s_{i, n}-n \varepsilon_{n}},
$$

where we used $x_{i, n}-x_{i+1, n}=-1 / n$.

Now for any $1 / 2>\delta>0$, there exists $N_{0} \in \mathbb{N}$ satisfying

$$
n \varepsilon_{n}=\frac{|g|_{\alpha}}{n^{\alpha-1}} \leq \delta \quad \text { for all } n \geq N_{0},
$$


in which case $s_{i, n}-n \varepsilon_{n} \geq s_{i, n}-\delta$, for all $n \geq N_{0}$, so that

$$
\frac{1}{s_{i, n}-n \varepsilon_{n}} \leq \frac{1}{s_{i, n}-\delta} \quad \text { for all } n \geq N_{0}
$$

This in combination with (3.10) yields

$$
t_{i, n} \leq \frac{s_{i, n}^{2}}{2\left(s_{i, n}-\delta\right)}<\left(\frac{1}{2}+\delta\right) s_{i, n}=\gamma s_{i, n} \quad \text { for all } n \geq N_{0},
$$

for $\gamma:=1 / 2+\delta \in(1 / 2,1)$, where we used $s_{i, n} \geq 1$ and $0<\delta<1 / 2$ to establish the second inequality. From this, in combination with (3.9), we get

$$
\begin{aligned}
s_{i+k, n}-t_{i, n} & \geq s_{i, n}-t_{i, n}-k \frac{C_{g}}{n^{\alpha-1}} \\
& \geq(1-\gamma) s_{i, n}-k \frac{C_{g}}{n^{\alpha-1}} \\
& \geq(1-\gamma)-k \frac{C_{g}}{n^{\alpha-1}} \geq 0
\end{aligned}
$$

for all $k$ satisfying $2 \leq k \leq 2 C_{r}$, where $C_{r}$ is the constant in (3.8). In this case, the interior of the circle $C_{i, n}$ and the points $\left\{p_{i+k, n}^{-}: 2 \leq k \leq 2 C_{r}\right\}$ are separated by the tangent of $C_{i, n}$ at $p_{i+1, n}^{-}$, see Figure 3 for illustration. But this implies that also the points $\left\{p_{i+k, n}^{+}: 2 \leq k \leq n C_{r}\right\}$ lie outside the circle $C_{i, n}$.

For the remaining cases, where $k>2 C_{r}$, we have $x_{i+k, n}-x_{i, n}=k / n>2 C_{r} / n$, in which case we can see that all points $\left\{p_{i+k, n}^{ \pm}: k>2 C_{r} / n\right\}$ do also lie outside $C_{i, n}$. Altogether, all points $\left\{p_{i+k, n}^{ \pm}: k=2, \ldots, n-i\right\}$ lie outside $C_{i, n}$.

In conclusion from cases $1 \mathrm{a}$ and $1 \mathrm{~b}$, we see that (for $n$ large enough) no point $\left\{p_{i, n}^{ \pm}: i=1, \ldots, n\right\}$ lies in the interior of the circle $C_{i, n}$. Only the three vertices $p_{i, n}^{-}, p_{i, n}^{+}, p_{i+1, n}^{-}$of triangle $T_{i, n}$ lie on the boundary of $C_{i, n}$. Therefore, triangle $T_{i, n}$ satisfies the Delaunay criterion, and so its three edges $\left[p_{i, n}^{-}, p_{i+1, n}^{-}\right],\left[p_{i, n}^{-}, p_{i, n}^{+}\right]$, and $\left[p_{i, n}^{+}, p_{i+1, n}^{-}\right]$are contained in the Delaunay triangulation $\mathcal{D}\left(Y_{n}\right)$.

Case 2. Suppose $s_{i, n}<0$. In this case, we can follow along the lines of exactly the same arguments as in case 1 , after a reflection of the $x$-coordinate about $x=1 / 2$, so that $g$ is replaced by $g(1-x)$, and $x_{i, n}$ is mapped onto $1-x_{i, n}$, for all $i=0, \ldots, n$, so that in particular the points $p_{i, n}^{ \pm}$are then in reverse order.

Case 3. Suppose $s_{i, n}=0$. In this case, $c_{i, n}=\left(x_{i, n}+1 /(2 n), g_{i, n}\right)$, so that $x_{i, n}<c_{x}<x_{i+1, n}$. Therefore, all points from $\left\{p_{k, n}^{ \pm}: k<i\right.$ or $\left.k>i+1\right\}$ lie outside the circle $C_{i, n}$. Moreover, since $g_{i, n}=g_{i+1, n}$, the four points $p_{i, n}^{ \pm}, p_{i+1, n}^{ \pm}$are the corners of the rectangle $R_{i, n}=\left[x_{i, n}, x_{i+1, n}\right] \times\left[g_{i, n}-\varepsilon_{n}, g_{i, n}+\varepsilon_{n}\right]$ centred at $c_{i, n}$. Therefore, all four corner points $p_{i, n}^{ \pm}, p_{i+1, n}^{ \pm}$lie on the boundary of circle $C_{i, n}$, so that all four edges $\left[p_{i, n}^{ \pm}, p_{i+1, n}^{ \pm}\right],\left[p_{i, n}^{-}, p_{i, n}^{+}\right]$, and $\left[p_{i+1, n}^{-}, p_{i+1, n}^{+}\right]$of rectangle $R_{i, n}$ are contained in the Delaunay triangulation $\mathcal{D}\left(Y_{n}\right)$ of $Y_{n}$.

Finally, to show that all edges $\left[p_{i, n}^{+}, p_{i+1, n}^{+}\right], i=0, \ldots, n$, are in $\mathcal{D}\left(Y_{n}\right)$, this can be done by similar arguments as in the above cases 1.-3., but now after a reflection of the $y$-coordinate about $y=1 / 2$, where $y$ is replaced by $1-y$.

Now we are in a position, where we can combine Proposition 3.2 with Lemma 3.4 to prove a result which is stronger than that in Proposition 3.2. We will so obtain 
asymptotic approximation rates as in Proposition 3.2, but now for linear splines over Delaunay triangulations rather than just over conformal triangulations.

Theorem 3.5. For $\alpha \in(1,2]$, let $f$ be an $\alpha$-horizon function. Then, there exist constants $C, M>0$ (independent of $N$ ), such that for any $N \in \mathbb{N}$ there is a Delaunay triangulation $\mathcal{D}_{N} \equiv \mathcal{D}\left(Y_{N}\right)$ with $\left|Y_{N}\right| \leq M \times N$ vertices satisfying

$$
\left\|f-f_{N}\right\|_{L^{2}[0,1]^{2}}^{2} \leq C N^{-\alpha},
$$

where $f_{N} \in \mathcal{S}_{\mathcal{D}_{N}}$ is the linear spline interpolant to $f$ at $Y_{N}$.

Proof. Let $Y_{N}$ be the vertex set in Lemma 3.4, whose size is $\left|Y_{N}\right|=2(N+1)+4$. Due to Lemma 3.4, all (horizontal) edges

$$
\left[p_{i, N}^{+}, p_{i+1, N}^{+}\right] \quad \text { and } \quad\left[p_{i, N}^{-}, p_{i+1, N}^{-}\right] \quad \text { for } i=0, \ldots, N-1
$$

and all (vertical) edges $\left[p_{i, N}^{-}, p_{i, N}^{+}\right], i=0, \ldots, N$, are contained in the Delaunay triangulation $\mathcal{D}_{N}$ of $Y_{N}$, for $N$ large enough.

We can complete the Delaunay triangulation $\mathcal{D}_{N}$ by first triangulating the area of the $\varepsilon_{N}$-corridor $K_{\varepsilon_{N}}$ (cf. the proof of Proposition 3.2) w.r.t. the Delaunay criterion. Note that $K_{\varepsilon_{N}}$ is given by the union of the parallelograms $Q_{i, N}=\left[p_{i, N}^{ \pm}, p_{i+1, N}^{ \pm}\right]$, for $i=0, \ldots, N-1$, and so the Delaunay triangulation of the corridor $K_{\varepsilon_{N}}$ can be obtained by the Delaunay triangulations of the parallelograms $Q_{i, N}$. This is followed by the construction of the Delaunay criterion in the complementary area $[0,1]^{2} \backslash K_{\varepsilon_{N}}$ which completes the Delaunay triangulation $\mathcal{D}_{N}$ of $Y_{N}$.

Now note that the linear spline interpolant $f_{N} \in \mathcal{S}_{\mathcal{D}_{N}}$ to $f$ at $Y_{N}$ coincides with $f$ on $[0,1]^{2} \backslash K_{\varepsilon_{N}}$, so that we obtain the desired asymptotic bound

$$
\left\|f-f_{N}\right\|_{L^{2}[0,1]^{2}}^{2} \leq C N^{-\alpha}
$$

by the following along the lines of our arguments in the proof of Proposition 3.2.

In conclusion, we have proved that approximation of any $\alpha$-horizon function, for $\alpha \in(1,2]$, (cf. Definition 3.1) by linear splines over locally adaptive triangulations leads to the asymptotic $N$-term approximation error

$$
\left\|f-f_{N}\right\|_{L^{2}[0,1]^{2}}^{2}=\mathcal{O}\left(N^{-\alpha}\right) \quad \text { for } N \rightarrow \infty
$$

when using conformal triangulations (Proposition 3.2) or when using Delaunay triangulations (Theorem 3.5). Recall that nonlinear $N$-term approximations by tensor product wavelets (1.2) can only achieve the suboptimal decay rate $\mathcal{O}\left(N^{-1 / 2}\right)$ [27].

To explain in which sense the achieved $N$-term approximation rates by Delaunay triangulations are optimal for $\alpha$-horizon functions, we rely on a concept from model selection theory, according to which it is important that the model sizes grow at a controlled rate (of at most polynomial growth). As proven in [18], there is no depth-search limited dictionary which can achieve a better decay rate than that in (3.11) for piecewise $\mathscr{C}^{\alpha}$ functions with singularities along $\mathscr{C}^{\alpha}$ curves.

To relate this statement to our setting, we remark that our results in Theorem 3.5 can be achieved by selecting the points $p_{i, N}^{ \pm}$, which are defining the Delaunay triangulations $\mathcal{D}\left(Y_{N}\right)$, from a fine grid whose size is of polynomial growth in $N$. To detail this, we consider a uniform grid with sampling size $1 / N^{\beta}$, where $\beta>\alpha$. Then, in particular, a representation for $f_{N}$ of the form (3.3) could also be obtained via a selection of $N$ functions from a dictionary $\mathcal{A}_{N}$, whose size is of polynomial growth 
in $N$, with maintaining the asymptotic error bound (3.11). Therefore, approximation by linear splines over Delaunay triangulations yields asymptotically optimal approximation rates among all depth-search limited dictionaries.

\section{Approximation of Regular Functions}

In the previous section, we have restricted our attention to the approximation of piecewise-affine horizon functions $f$ with singularities along $\alpha$-Hölder smooth boundary curves $g \in \mathscr{C}^{\alpha}[0,1]$. In that case, our approximation scheme, relying on linear splines over anisotropic (Delaunay) triangulations, is adapted to the smoothness $\alpha$ of the horizon boundary $g$, whereas the regularity of the target function $f$ away from the horizon boundary does not take impact on the adaptivity of the triangular mesh.

In this section, we turn to the approximation of regular functions, where a regular function $f$ is an element in a Sobolev space $W^{\alpha, p}\left([0,1]^{2}\right)$, for $\alpha \in(0,2]$ and $p \geq 1$, where $\alpha>2 / p-1$. In this case, $W^{\alpha, p}\left([0,1]^{2}\right)$ is embedded in $L^{2}\left([0,1]^{2}\right)$ (see e.g. [20, Subsection 2.5.1]), but does not lie on the $L^{2}\left([0,1]^{2}\right)$ embedding line. For the sake of brevity, we will then say that $W^{\alpha, p}\left([0,1]^{2}\right)$ lies above the $L^{2}$-embedding line.

We will essentially adapt our approximation scheme to the regularity of $f$. Note that regular functions are isotropic and, moreover, they can be characterised by the asymptotic behaviour of their wavelet coefficients. We remark that the class of regular functions, being investigated in this section, form a rather large subset in the linear space of all functions which can be approximated by classical nonlinear (tensor product) wavelet approximations at a decay rate $\mathcal{O}\left(N^{-\alpha / 2}\right)$, for $N \rightarrow \infty$, cf. the discussion at the end of this section.

4.1. Optimal Approximation with Quadtree Partitions. To detail our analysis, we recall a classical result by Birman-Solomjak [4] concerning the approximation of regular functions by piecewise-affine functions over quadtree partitions of $[0,1]^{2}$. Before doing so, we first give a formal definition for quadtree partitions.

Definition 4.1. A finite set $\mathcal{Q}=\left\{Q_{n}\right\}_{n}$ of pairwise distinct closed dyadic squares

$$
Q_{n}=\left[\frac{i}{2^{k}}, \frac{i+1}{2^{k}}\right] \times\left[\frac{j}{2^{k}}, \frac{j+1}{2^{k}}\right], \quad \text { for } i, j \in\left\{0, \ldots, 2^{k}-1\right\} \text { and } k \in \mathbb{N},
$$

whose union is $[0,1]^{2}$, is called a quadtree partition of the unit square $[0,1]^{2}$.

For the purpose of illustration, Figure $4(\mathrm{a})$ shows one quadtree partition of $[0,1]^{2}$.

Theorem 4.2 (Birman-Solomjak). Let $\alpha \in(0,2]$ and $p \geq 1$ satisfy $\alpha>2 / p-1$, so that $W^{\alpha, p}\left([0,1]^{2}\right)$ lies strictly above the $L^{2}$-embedding line. Further suppose $f \in W^{\alpha, p}\left([0,1]^{2}\right)$. Then there exists a constant $C>0$ (independent of $N$ ), such that for any $N \in \mathbb{N}$ there is a quadtree partition $\mathcal{Q}_{N}$ of $[0,1]^{2}$ with $\left|\mathcal{Q}_{N}\right| \leq N$ leaves satisfying

$$
\left\|f-f_{N}\right\|_{L^{2}\left([0,1]^{2}\right)}^{2} \leq C N^{-\alpha},
$$

where $f_{N}:=\Pi_{\mathcal{Q}_{N}} f$ is the orthogonal $L^{2}$-projection of $f$ onto the space of piecewise affine-linear (not necessarily continuous) functions over the quadtree partition $\mathcal{Q}_{N}$. 
We remark that the proof of Birman-Solomjak is constructive. In particular, an explicit algorithmic construction of a quadtree partition $\mathcal{Q}_{N}$ satisfying the error estimate (4.1) is provided in [4]. But $\mathcal{Q}_{N}$ does not necessarily minimize the approximation error in (4.1) among all quadtree partitions $\mathcal{Q}$ of size $|\mathcal{Q}| \leq N$. This may affect the size of the constant $C$ but not the asymptotic decay rate in (4.1).

4.2. Optimal Approximation with Delaunay Triangulations. Now let us turn to the approximation of regular functions by adaptive linear splines over anisotropic Delaunay triangulations. On the basis of the construction by BirmanSolomjak, we can construct a sequence of Delaunay triangulations $\mathcal{D}_{N}$, such that the corresponding sequence of orthogonal $L^{2}$-projections $\Pi_{\mathcal{D}_{N}} f$ of $f$ onto the space $\mathcal{S}_{\mathcal{D}_{N}}$ of linear splines over $\mathcal{D}_{N}$ achieves the same approximation rate as the sequence of functions $\Pi_{\mathcal{Q}_{N}} f$ from the Birman-Solomjak Theorem.

Corollary 4.3. Let $\alpha \in(0,2]$ and $p \geq 1$ satisfy $\alpha>2 / p-1$, so that $W^{\alpha, p}\left([0,1]^{2}\right)$ lies strictly above the $L^{2}$-embedding line. Suppose $f \in W^{\alpha, p}\left([0,1]^{2}\right)$. Then there exist constants $C, M>0$ (independent of $N$ ), such that for any $N \in \mathbb{N}$ there is a Delaunay triangulation $\mathcal{D}_{N}$ of size $\left|\mathcal{D}_{N}\right| \leq M \times N$ satisfying

$$
\left\|f-\Pi_{\mathcal{D}_{N}} f\right\|_{L^{2}\left([0,1]^{2}\right)}^{2} \leq C N^{-\alpha}
$$

where $\Pi_{\mathcal{D}_{N}} f$ is the orthogonal $L^{2}$-projection of $f$ onto $\mathcal{S}_{\mathcal{D}_{N}}$.

Proof. We split the proof into three steps.

Step 1. Let $\left\{\mathcal{Q}_{N}\right\}_{N}$ denote the sequence of quadtree partitions from the BirmanSolomjak Theorem, satisfying (4.1). By $V_{\mathcal{Q}_{N}}=\left\{\left(x_{m}, y_{m}\right)\right\}_{m=1, \ldots, M}$ we denote, for any $N \in \mathbb{N}$, the vertex set of $\mathcal{Q}_{N}$, comprising all $M$ vertices from the $\left|\mathcal{Q}_{N}\right| \leq N$ quadtree leaves, so that $M \leq 4 \times N$.

Next we associate, for some (sufficiently small) $\varepsilon>0$, the vertex set $V_{\mathcal{Q}_{N}}$ with the perturbed planar point set

$$
V_{M, \varepsilon}=\left(\left\{\left(x_{m} \pm \varepsilon, y_{m} \pm \varepsilon\right): m=1, \ldots, M\right\} \cup\{(0,0),(0,1),(1,0),(1,1)\}\right) \cap[0,1]^{2} .
$$

For illustration, Figure 4 (a) shows one example for a quadtree partition $\mathcal{Q}_{N}$ with vertex set $V_{\mathcal{Q}_{N}}$. Its associated partition, resulting from the perturbed point set $V_{M, \varepsilon}$, is shown in Figure 4 (b). Note that $\left|V_{M, \varepsilon}\right| \leq 4 \times M \leq 16 \times N$.

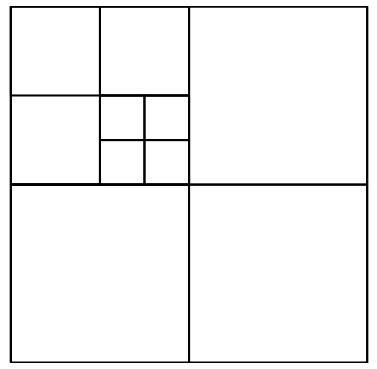

(a)

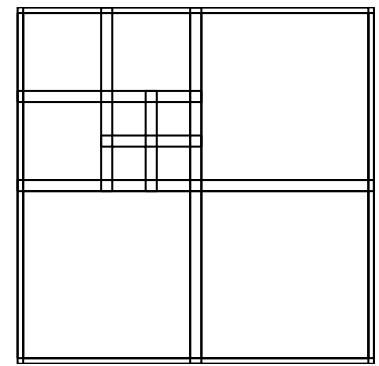

(b)

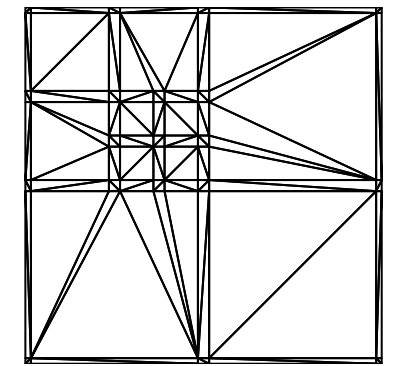

(c)

Figure 4. (a) Quadtree partition $\mathcal{Q}_{N}$ of the unit square $[0,1]^{2}$, with vertex set $V_{\mathcal{Q}_{N}}$, (b) Associated partition from perturbed vertex set $V_{M, \varepsilon}$, (c) Delaunay triangulation $\mathcal{D}\left(V_{M, \varepsilon}\right)$. 
Step 2. Now we construct the Delaunay triangulation $\mathcal{D}\left(V_{M, \varepsilon}\right)$ of the perturbed point set $V_{M, \varepsilon}$. To this end, we partition the domain $[0,1]^{2}$ into a set of disjoint areas, $M$ small subsquares $s_{m}, N$ large subsquares $S_{n}$, and $K$ anisotropic rectangles $R_{k}$, so that

$$
[0,1]^{2}=\left(\bigcup_{m=1}^{M} s_{m}\right) \bigcup\left(\bigcup_{n=1}^{N} S_{n}\right) \bigcup\left(\bigcup_{k=1}^{K} R_{k}\right)
$$

where each small subsquare $s_{m} \subset[0,1]^{2}$ is defined as

$$
s_{m}=\operatorname{conv}\left\{\left(x_{m} \pm \varepsilon, y_{m} \pm \varepsilon\right)\right\} \cap[0,1]^{2} \quad \text { for } m=1, \ldots, M .
$$

As for the subsquares $S_{n}$, recall from Definition 4.1 that any element $Q_{n} \in \mathcal{Q}_{N}$ of the Birman-Solomjak quadtree partition $\mathcal{Q}_{N}$ has the form

$$
Q_{n}=\left[\frac{i}{2^{k}}, \frac{i+1}{2^{k}}\right] \times\left[\frac{j}{2^{k}}, \frac{j+1}{2^{k}}\right] \quad \text { for some } 0 \leq i, j, k \in \mathbb{N} .
$$

We define any large subsquare $S_{n} \subset Q_{n}$ as

$$
S_{n}=\left[\frac{i}{2^{k}}+\varepsilon, \frac{i+1}{2^{k}}-\varepsilon\right] \times\left[\frac{j}{2^{k}}+\varepsilon, \frac{j+1}{2^{k}}-\varepsilon\right] \quad \text { for } n=1, \ldots, N .
$$

Now note that the complement

$$
[0,1]^{2} \backslash\left(\left(\bigcup_{m=1}^{M} s_{m}\right) \bigcup\left(\bigcup_{n=1}^{N} S_{n}\right)\right)
$$

can be partitioned by $K=4 \times N$ long and thin (anisotropic) rectangles with pairwise disjoint interior. We denote these rectangles as $R_{k}, k=1, \ldots, K$. Figure 4 (b) shows one example for such a decomposition of $[0,1]^{2}$ into a union of small subsquares $s_{m}$, large subsquares $S_{n}$ and anisotropic rectangles $R_{k}$ with pairwise disjoint interior.

Next, we observe that for each small subsquare $s_{m}$ lying in the interior of the unit square $[0,1]^{2}$, and for sufficiently small $\varepsilon>0$, the four pairs of its vertices,

$$
\begin{array}{lll}
\left(x_{m}-\varepsilon, y_{m}-\varepsilon\right) & \text { and } & \left(x_{m}+\varepsilon, y_{m}-\varepsilon\right) \\
\left(x_{m}+\varepsilon, y_{m}-\varepsilon\right) & \text { and } & \left(x_{m}+\varepsilon, y_{m}+\varepsilon\right) \\
\left(x_{m}+\varepsilon, y_{m}+\varepsilon\right) & \text { and } & \left(x_{m}-\varepsilon, y_{m}+\varepsilon\right) \\
\left(x_{m}-\varepsilon, y_{m}+\varepsilon\right) & \text { and } & \left(x_{m}-\varepsilon, y_{m}-\varepsilon\right)
\end{array}
$$

are Voronoi neighbours in the Voronoi diagram $\mathcal{V}\left(V_{M, \varepsilon}\right)$ of $V_{M, \varepsilon}$, respectively. Likewise, for each subsquare $s_{m}$ lying adjacent to the boundary of the unit square $[0,1]^{2}$, its corresponding four vertex pairs of its four vertices are Voronoi neighbours in $\mathcal{V}\left(V_{M, \varepsilon}\right)$.

Due to the duality of the Voronoi diagram $\mathcal{V}\left(V_{M, \varepsilon}\right)$ and the Delaunay triangulation $\mathcal{D}\left(V_{M, \varepsilon}\right)$, all four edges of any subsquare $s_{m}$ are edges in the Delaunay triangulation $\mathcal{D}\left(V_{M, \varepsilon}\right)$, for $m=1, \ldots, M$. Likewise, all four edges of any rectangle $R_{k}$ are edges in $\mathcal{D}\left(V_{M, \varepsilon}\right)$, for $k=1, \ldots, K$.

To complete the Delaunay triangulation $\mathcal{D}\left(V_{M, \varepsilon}\right)$ of $V_{M, \varepsilon}$, it remains to triangulate the subsquares $s_{m}$ and $S_{n}$, as well as the rectangles $R_{k}$. For the small subsquares $s_{m}$ and the rectangles $R_{k}$, this can be done by splitting each subdomain, $s_{m}$ or $R_{k}$, across any of its two diagonals. But note that in this case their Delaunay triangulation is not unique, since any of $s_{m}$ or $R_{k}$ could also be split across the other diagonal, respectively. 
As regards the large subsquares $S_{n}$, note that any $S_{n}$ may contain, besides its four corner points, further points from $V_{M, \varepsilon}$ on its boundary. Therefore, the triangulation of $S_{n}$ is accomplished by triangulating the point set $S_{n} \cap V_{M, \varepsilon}$ according to the Delaunay criterion. Figure 4 (c) shows one example for a Delaunay triangulation $\mathcal{D}\left(V_{M, \varepsilon}\right)$ of a perturbed vertex set $V_{M, \varepsilon}$.

Step 3. Finally, let $f_{N} \in \mathcal{S}_{\mathcal{D}_{N}}, \mathcal{D}_{N}=\mathcal{D}\left(V_{M, \varepsilon}\right)$, be the unique linear spline function which interpolates the piecewise affine-linear Birman-Solomjak function $\Pi_{\mathcal{Q}_{N}} f$ in (4.1) at the vertices $V_{M, \varepsilon}$. Note that $f_{N}$ coincides with $\Pi_{\mathcal{Q}_{N}} f$ on each large subsquare $S_{n}$, and therefore

$$
\left\|f_{N}-\Pi_{\mathcal{Q}_{N}} f\right\|_{L^{2}\left([0,1]^{2}\right)}^{2}=\sum_{m=1}^{M}\left\|f_{N}-\Pi_{\mathcal{Q}_{N}} f\right\|_{L^{2}\left(s_{m}\right)}^{2}+\sum_{k=1}^{K}\left\|f_{N}-\Pi_{\mathcal{Q}_{N}} f\right\|_{L^{2}\left(R_{k}\right)}^{2} .
$$

Now we can bound the $L^{2}$-error over any small square $s_{m}$ by

$$
\left\|f_{N}-\Pi_{\mathcal{Q}_{N}} f\right\|_{L^{2}\left(s_{m}\right)}^{2} \leq C\left\|\Pi_{\mathcal{Q}_{N}} f\right\|_{\infty}^{2} \int_{s_{m}} d x d y=C\left\|\Pi_{\mathcal{Q}_{N}} f\right\|_{\infty}^{2} 4 \varepsilon^{2}
$$

for some constant $C>0$ independent of $N$. Likewise, we can bound the $L^{2}$-error over any rectangle $R_{k}$ by

$$
\left\|f_{N}-\Pi_{\mathcal{Q}_{N}} f\right\|_{L^{2}\left(R_{k}\right)}^{2} \leq C\left\|\Pi_{\mathcal{Q}_{N}} f\right\|_{\infty}^{2} \int_{R_{k}} d x d y \leq C\left\|\Pi_{\mathcal{Q}_{N}} f\right\|_{\infty}^{2} 2 \varepsilon
$$

for some constant $C>0$ independent of $N$. This then yields the error estimate

$$
\begin{aligned}
\left\|f-\Pi_{\mathcal{D}_{N}} f\right\|_{L^{2}\left([0,1]^{2}\right)}^{2} & \leq\left\|f-f_{N}\right\|_{L^{2}\left([0,1]^{2}\right)}^{2} \\
& \leq\left\|f-\Pi_{\mathcal{Q}_{N}} f\right\|_{L^{2}\left([0,1]^{2}\right)}^{2}+\left\|f_{N}-\Pi_{\mathcal{Q}_{N}} f\right\|_{L^{2}\left([0,1]^{2}\right)}^{2} \\
& \leq C N^{-\alpha}+C \varepsilon,
\end{aligned}
$$

for arbitrarily small $\varepsilon>0$. We let $\varepsilon=N^{-\alpha}$ to complete our proof.

4.3. Concluding Remarks, Comparison with Wavelets, and Optimality. Let us finally make a few remarks concerning the result of our Corollary 4.3.

Although the proof by Birman-Solomjak of Theorem 4.2 is constructive, it does not provide a sequence of optimal quadtree partitions, $\left\{\mathcal{Q}_{N}^{*}\right\}$, satisfying

$$
\left\|f-\Pi_{\mathcal{Q}_{N}^{*}} f\right\|_{L^{2}\left([0,1]^{2}\right)}=\inf _{\mathcal{Q}_{N}}\left\|f-\Pi_{\mathcal{Q}_{N}} f\right\|_{L^{2}\left([0,1]^{2}\right)} .
$$

Moreover, since our construction in Corollary 4.3 relies on quadtree partitions, the Delaunay triangulations $\mathcal{D}_{N}$ in Corollary 4.3 are in general not optimal either. To improve on the quality of the Delaunay triangulations $\mathcal{D}_{N}$, as output by our construction in Corollary 4.3, one should essentially avoid long thin triangles, as they are resulting from the splitting of the long thin rectangles $R_{k}$ (cf. the proof of Corollary 4.3). Instead, one should rather work with adaptive Delaunay triangulations containing isotropic triangles, since the target function $f$ is assumed to be regular. In that case, however, it is much harder to prove optimal rates for asymptotic $N$-term approximations, where the technical difficulties are mainly due to the Delaunay criterion.

But our greedy approximation algorithm, adaptive thinning $[11,13]$, achieves to construct a sequence of anisotropic Delaunay triangulations $\left\{\mathcal{D}_{N}^{*}\right\}_{N}$, whose corresponding linear spline interpolants $f_{N}^{*} \in \mathcal{D}_{N}^{*}$ improve the approximation quality of the interpolants $f_{N} \in \mathcal{D}_{N}$ output in Corollary 4.3. In fact, as supported by 
our numerical results, the smallest constant $M$ in Corollary 4.3, reflecting the data size of the Delaunay triangulations $\mathcal{D}_{N}^{*}$, can, at equal approximation error and in comparison with the Delaunay triangulations $\mathcal{D}_{N}$ in Corollary 4.3 , be reduced quite significantly.

We may be able to show that for the piecewise affine-linear target functions, i.e., the Birman-Solomjak functions $\Pi_{\mathcal{Q}_{N}} f$ in (4.1), adaptive thinning outputs a sequence of Delaunay triangulations $\left\{\mathcal{D}_{N}^{*}\right\}_{N}$ which are "close" to those Delaunay triangulations $\mathcal{D}_{N}$ in the proof of Corollary 4.3, along with a sequence of corresponding linear spline interpolants $f_{N}^{*} \in \mathcal{D}_{N}^{*}$ that approximate $f$ at the same rate as the functions $\Pi_{\mathcal{Q}_{N}} f$. We prefer to defer this rather delicate point to future work.

By Corollary 4.3, any regular function $f \in W^{\alpha, p}\left([0,1]^{2}\right), \alpha>2 / p-1$, can be approximated by linear splines over Delaunay triangulations at an $N$-term approximation rate of $N^{-\alpha / 2}$. We remark that this approximation rate is at least as good as the approximation rate which can be achieved by nonlinear wavelet approximation. In fact, nonlinear wavelet approximation to $f$ may only be superior in situations, where $f$ does not belong to any of the Sobolev spaces $W^{\alpha, p}\left([0,1]^{2}\right)$ covered by our Corollary 4.3, but lies in the wavelet approximation space $B_{\tau, \tau}^{\alpha}\left([0,1]^{2}\right)$, where $1 / \tau=1 / p+\alpha / 2$, cf. equation (7.41) and Remark 7.6 in [14].

Finally, we remark that the asymptotic decay rate of wavelet representations is optimal for the Sobolev spaces $W^{\alpha, p}\left([0,1]^{2}\right)$ which are considered in Corollary 4.3 (see, e.g., [17] and references therein). This implies that the Birman-Solomjak quadtree representations (Theorem 4.2) and those of Delaunay triangulations (as constructed in the proof Corollary 4.3) provide asymptotically optimal decay rates for the class of regular functions $W^{\alpha, p}\left([0,1]^{2}\right)$, where $\alpha>2 / p-1$.

\section{Approximation of Piecewise Regular Horizons}

We remark that the utilized regularity concepts of Sections 3 and 4 are of fundamental difference. While the approximation of regular functions (as in Section 4) could be covered by classical linear approximation methods (e.g. with wavelet orthonormal bases), the horizon functions of Section 3 have singularities which are concentrated along a regular curve. For the latter class of target functions, optimal approximation rates can only be achieved by anisotropic methods, as considered in this paper.

In this section, we combine the two different regularity concepts from previous Sections 3 and 4 for the purpose of approximating generalized horizons, i.e., piecewise regular horizon functions. But this requires a very careful treatment in regions close to the horizon boundary. To handle the resulting technical problems, we work with suitable extension operators, whereby we need to restrict ourselves to the special case, where $p=2$ and $\alpha \in(1,2)$, to approximate piecewise regular functions from the regularity class $W^{\alpha, 2}\left([0,1]^{2}\right)$.

5.1. Generalized Horizons. Let us first define the class of generalized horizons.

Definition 5.1. For $\alpha \in(1,2)$ and $g \in \mathscr{C}^{\alpha}([0,1])$, let

$$
\Omega^{+}=\left\{(x, y) \in(0,1)^{2}: y>g(x)\right\} \text { and } \Omega^{-}=\left\{(x, y) \in(0,1)^{2}: y<g(x)\right\}
$$

denote the hypograph and epigraph of $g$ on $(0,1)^{2}$. A function $f \in L^{2}\left([0,1]^{2}\right)$ is then said to be a generalized $\alpha$-horizon, iff each of its restrictions $\left.f\right|_{\Omega^{ \pm}}$to $\Omega^{ \pm}$lies in 
$W^{\alpha, 2}\left(\Omega^{ \pm}\right)$, where $W^{\alpha, 2}\left(\Omega^{ \pm}\right)$are the usual Sobolev spaces of regularity $\alpha$ w.r.t. the $L^{2}$-norm on $\Omega^{ \pm}$. We collect all generalized $\alpha$-horizons in the function class

$$
\mathscr{H}^{\alpha, 2}\left([0,1]^{2}\right)=\left\{f \in L^{2}\left([0,1]^{2}\right):\left.f\right|_{\Omega^{+}} \in W^{\alpha, 2}\left(\Omega^{+}\right) \text {and }\left.f\right|_{\Omega^{-}} \in W^{\alpha, 2}\left(\Omega^{-}\right)\right\} .
$$

Note that the set $\mathscr{H}^{\alpha, 2}\left([0,1]^{2}\right)$ is well-defined. Moreover, the open domains $\Omega^{+}$ and $\Omega^{-}$are simply connected and their boundaries are closed Jordan curves.

5.2. Optimal Approximation of Generalized Horizons. Now we approximate generalized $\alpha$-horizons by linear splines over anisotropic Delaunay triangulations. To this end, we use specific ingredients from our analysis of the previous two sections. In particular, we will construct point sets $X_{N}$, such that their Delaunay triangulations $\mathcal{D}\left(X_{N}\right)$ lead (for sufficiently large $N$ ) to a sequence of linear spline interpolants $f_{N} \in \mathcal{S}_{\mathcal{D}\left(X_{N}\right)}$ satisfying

$$
\left\|f-f_{N}\right\|_{L^{2}([0,1])}^{2} \leq C N^{-\alpha}
$$

where, moreover, the number of triangles in $\mathcal{D}\left(X_{N}\right)$ can be bounded above by $\left|\mathcal{D}\left(X_{N}\right)\right| \leq M \times N$, for some $M>0$, where $M$ does not depend on $N$.

Now recall the point sets $Y_{n}$ from Lemma 3.4. To discuss the construction of suitable points sets $Y_{N}$, we restrict ourselves, for the sake of simplicity and without loss of generality, to the cases where $\sqrt{N} \in \mathbb{N}$. Let

$$
U_{N}:=\left\{\left(\frac{i}{\sqrt{N}}, \frac{j}{\sqrt{N}}\right): 0 \leq i, j \leq \sqrt{N}\right\} \quad \text { for } \sqrt{N} \in \mathbb{N}
$$

be a set of uniformly sampled points in $[0,1]^{2}$ and, moreover, let

$$
G_{N}:=\left\{z \in[0,1]^{2}: \operatorname{dist}(z, \operatorname{graph}(g)) \leq \frac{2 C_{r}}{N}\right\}
$$

denote the set of all points in the unit square whose distance to the graph of $g$,

$$
\operatorname{graph}(g):=\{(x, g(x)): x \in[0,1]\},
$$

is at most $2 C_{r} / N$, where $C_{r}$ is the same constant as in (3.8). Finally, we define the point sets

$$
X_{N}:=Y_{N} \cup\left(U_{N} \backslash G_{N}\right),
$$

whose following properties of their Delaunay triangulations $\mathcal{D}\left(X_{N}\right)$ will be useful in our subsequent analysis.

Lemma 5.2. The Delaunay triangulation $\mathcal{D}\left(X_{N}\right)$ of the point set $X_{N}$ in (5.2) satisfies the following properties, provided that $N \in \mathbb{N}$ is large enough.

(a) All line segments $\left[p_{i, N}^{+}, p_{i+1, N}^{+}\right]$and $\left[p_{i, N}^{-}, p_{i+1, N}^{-}\right]$are edges in $\mathcal{D}\left(X_{N}\right)$.

(b) The diameter of any triangle $T \in \mathcal{D}\left(X_{N}\right)$ can be bounded above by

$$
\operatorname{diam}(T) \leq 2 \sqrt{2 / N}
$$

(c) The number of triangles in $\mathcal{D}\left(X_{N}\right)$ is, for some $M \in \mathbb{N}$, bounded by

$$
\left|\mathcal{D}\left(X_{N}\right)\right| \leq M \times N \quad \text { for all } N \in \mathbb{N}
$$

Proof. (a) By definition, any point in $U_{N} \backslash G_{N}$ is of distance at least $2 C_{r} / N$ away from graph $(g)$. Note that the distance between any point in the interior of any circle $C_{i, N}$ to its nearest point in $\operatorname{graph}(g)$ is smaller than the diameter $\operatorname{diam}\left(C_{i, N}\right)=$ $2 r_{i, N}$ of $C_{i, N}$. Therefore, due to the uniform bound on the radii $r_{i, N}$ in $(3.8)$, no point from $U_{N} \backslash G_{N}$ is contained in the interior of a circle $C_{i, N}$, provided that $N$ 
is large enough. Using similar arguments as in the proof of Lemma 3.4, this shows that all line segments $\left[p_{i, N}^{+}, p_{i+1, N}^{+}\right]$and $\left[p_{i, N}^{-}, p_{i+1, N}^{-}\right], i=0, \ldots, N-1$, are edges in $\mathcal{D}\left(X_{N}\right)$.

(b) Note that

$$
\frac{C_{r}}{N}<\frac{1}{\sqrt{N}}
$$

holds for $N$ large enough, which we tacitly assume from now. In this case, the assertion in (b) is obvious for any triangle $T$ containing only vertices in $Y_{N}$, or, for triangles $T$ containing only vertices in $U_{N} \backslash G_{N}$.

Let us now consider a triangle $T$ with vertices in both sets, $Y_{N}$ and $U_{N} \backslash G_{N}$. Moreover, suppose that the circumcircle $C_{T}$ of $T$ is strictly larger than $2 \sqrt{2 / N}$. Then, due to (5.3), $C_{T}$ contains at least three vertices from $X_{N}$. But this violates the Delaunay criterion, which is in contradiction to our assumption on $\mathcal{D}\left(X_{N}\right)$.

(c) Note that the size $\left|U_{N}\right|$ of the regular point set $U_{N}$ is $(\sqrt{N}+1)^{2}$, and so $\left|U_{N} \backslash G_{N}\right| \leq(\sqrt{N}+1)^{2}$. Moreover, the point set $Y_{N}$, as defined in Lemma 3.4, contains $2(N+1)+4$ points. But this implies that the size of $X_{N}$ can be bounded above by a constant multiple of $N$, in particular by $\left|X_{N}\right| \leq 4 \times N$ for sufficiently large $N$. In this case, and by using the Euler polyhedron formula, we can also bound the number of triangles in $\mathcal{D}\left(X_{N}\right)$ by an integer multiple of $N$, so that $\left|\mathcal{D}\left(X_{N}\right)\right| \leq M \times N$ for some $M>0$ independent of $N$.

For the purpose of illustration, Figure 5 shows one example for a Delaunay triangulation $\mathcal{D}\left(X_{N}\right)$, according to our above construction. Note that the triangulation $\mathcal{D}\left(X_{N}\right)$ is not adaptive in the two regions $\Omega^{ \pm} \backslash G_{N}$, where $f \in W^{\alpha, 2}\left(\Omega^{ \pm}\right)$is regular. This is in contrast to the adaptive partition of the domain $[0,1]^{2}$ by anisotropic quadtrees (Birman-Solomjak, Theorem 4.2) or by anisotropic Delaunay triangulations (in our Corollary 4.3). But the above construction of $X_{N}$ serves to simplify our analysis in the proof of Theorem 5.3.

Now we are in a position, where we can prove the main result of this section.

Theorem 5.3. For $\alpha \in(1,2)$, let $f$ be a generalized $\alpha$-horizon. Then, there exist constants $C, M>0$ (independent of $N$ ), such that for any $N \in \mathbb{N}$ there is a Delaunay triangulation $\mathcal{D}_{N}$ with $\left|\mathcal{D}_{N}\right| \leq M \times N$ triangles satisfying

$$
\left\|f-f_{N}\right\|_{L^{2}\left([0,1]^{2}\right)}^{2} \leq C N^{-\alpha},
$$

where $f_{N}:=\Pi_{\mathcal{D}_{N}} f$ is the orthogonal $L^{2}$-projection of $f$ onto $\mathcal{S}_{\mathcal{D}_{N}}$.

Proof. Since the boundary horizon $g$ of $f$ is in $\mathscr{C}^{\alpha}([0,1])$, with $\alpha>1, g$ is a Lipschitz function. Therefore, both domains $\Omega^{+}$and $\Omega^{-}$are Lipschitz. This allows us to apply the Stein extension theorem (see [1, Theorem 5.24]), which implies that for any $m \in \mathbb{N}$ there are strong and bounded total extension operators

$$
E_{m}^{ \pm}: W^{m, 2}\left(\Omega^{ \pm}\right) \rightarrow W^{m, 2}\left(\mathbb{R}^{2}\right) .
$$

We apply classical operator theory (see e.g. [3, Corollary 4.13]) to interpolate $E_{m}^{ \pm}$, $m=1,2$, for $\alpha \in(1,2)$, where the resulting interpolation operators

$$
E_{\alpha}^{ \pm}: W^{\alpha, 2}\left(\Omega^{ \pm}\right) \rightarrow W^{\alpha, 2}\left(\mathbb{R}^{2}\right)
$$

are bounded on the intermediate Sobolev spaces of order $\alpha \in(1,2)$. 


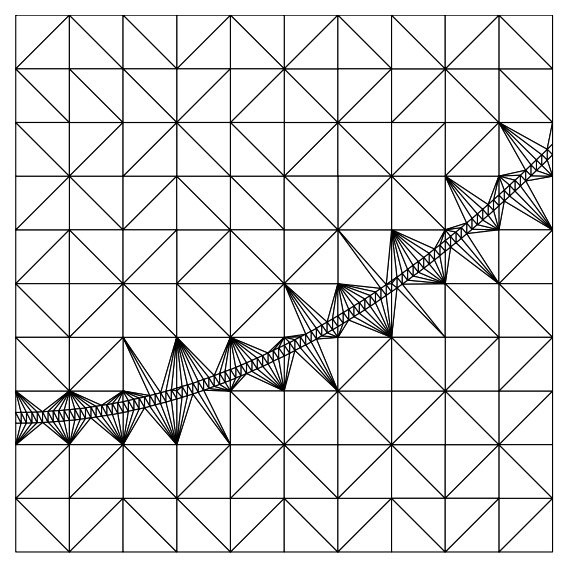

Figure 5. Approximation of a generalized horizon $f$. The Delaunay triangulation $\mathcal{D}\left(X_{N}\right)$ of the point set $X_{N}=Y_{N} \cup\left(U_{N} \backslash G_{N}\right)$ in (5.2) is shown. Note that the vertices of the squares in the regular part of $f$ are a sufficient distance away from the horizon boundary $g$. Moreover, any triangle in $\mathcal{D}\left(X_{N}\right)$ is contained in a square of edge length $2 / \sqrt{N}$.

Now, in order to prove the error estimate in (5.4) we use techniques similar to those in Section 4. In particular, we rely on the Birman-Solomjak bounds (4.1) and on our error estimates (4.2). As in our proof of Corollary 4.3, we work with perturbed point sets

$$
X_{N, \varepsilon}:=Y_{N} \cup\left(U_{N, \varepsilon} \backslash G_{N}\right)
$$

being associated with the point sets $X_{N}$ in (5.2), where we do only perturb the grid points in $U_{N}$, but not the points in $Y_{N}$.

Moreover, we let $\mathcal{D}_{N, \varepsilon}:=\mathcal{D}\left(X_{N, \varepsilon}\right)$ be the Delaunay triangulation of $X_{N, \varepsilon}$ and

$$
\Omega_{N, \varepsilon}^{ \pm}:=\bigcup\left\{T \in \mathcal{D}_{N, \varepsilon}: T \subset \Omega^{ \pm}\right\} \subset \Omega^{ \pm}
$$

denotes the union of all triangles in $\mathcal{D}_{N, \varepsilon}$ that are contained in $\Omega^{ \pm}$. Next we decompose the domain $[0,1]^{2}$ into three subdomains with pairwise disjoint interior,

$$
[0,1]^{2}=K_{\varepsilon_{N}} \cup \Omega_{N, \varepsilon}^{+} \cup \Omega_{N, \varepsilon}^{-},
$$

where $K_{\varepsilon_{N}}=[0,1]^{2} \backslash \Omega_{N, \varepsilon}^{ \pm}$is, for $\varepsilon_{N}>0$, an $\varepsilon_{N^{-}}$-corridor around $g$ of the form (3.4).

Now let $f_{N, \varepsilon}:=\Pi_{\mathcal{D}_{N, \varepsilon}} f$ denote the orthogonal $L^{2}$-projection of $f$ onto $\mathcal{S}_{\mathcal{D}\left(X_{N, \varepsilon}\right)}$. We split the $L^{2}$-approximation error between $f$ and $f_{N, \varepsilon}$ as

$$
\begin{aligned}
\left\|f-f_{N, \varepsilon}\right\|_{L^{2}\left([0,1]^{2}\right)}^{2}= & \left\|f-f_{N, \varepsilon}\right\|_{L^{2}\left(K_{\varepsilon_{N}}\right)}^{2} \\
& +\left\|f-f_{N, \varepsilon}\right\|_{L^{2}\left(\Omega_{N, \varepsilon}^{+}\right)}^{2}+\left\|f-f_{N, \varepsilon}\right\|_{L^{2}\left(\Omega_{N, \varepsilon}^{-}\right)}^{2} .
\end{aligned}
$$

Note that the first term in the right hand side of (5.5) can be bounded above by

$$
\left\|f-f_{N, \varepsilon}\right\|_{L^{2}\left(K_{\varepsilon_{N}}\right)}^{2} \leq C \cdot\|f\|_{L^{\infty}\left([0,1]^{2}\right)}^{2} \cdot\left|K_{\varepsilon_{N}}\right| \leq C N^{-\alpha},
$$

where $\left|K_{\varepsilon_{N}}\right|$ is the area of $K_{\varepsilon_{N}}$ and $C>0$ is some constant independent of $N$ (cf. our proof of Proposition 3.2). 
In order to obtain upper bounds for the other two terms in (5.5), we consider using, for each of the two extensions $E_{\alpha}^{ \pm} f:[0,1]^{2} \rightarrow \mathbb{R}$ (here restricted to $[0,1]^{2}$ ), their corresponding sequence of Birman-Solomjak approximations $\Pi_{\mathcal{Q}_{N}} E_{\alpha}^{ \pm} f$ (from Theorem 4.2). Recall that the Birman-Solomjak bounds (4.1) are relying, for the general case of approximating functions from $W^{\alpha, p}\left([0,1]^{2}\right)$, for $\alpha>2 / p-1$, on adaptive quadtree partitions.

We remark that in the situation of this proof, where we are concerned with the special case $p=2$, i.e., approximation in $W^{\alpha, 2}\left([0,1]^{2}\right)$ for $\alpha \in(1,2)$, we obtain error bounds of the form (4.1) for non-adaptive quadtree partitions $\mathcal{Q}_{N}$ of $[0,1]^{2}$ (see [4]), consisting of quadtree elements, each of whose diameter is bounded above by $C / \sqrt{N}$, for some constant $C>0$ independent of $N$ (cf. Figure 5 for illustration).

By following along the lines of our construction in the proof of Corollary 4.3, we obtain, for each of the two extensions $E_{\alpha}^{ \pm} f$, a corresponding sequence of approximations $f_{N, \epsilon}^{ \pm}=\Pi_{\mathcal{D}_{N, \varepsilon}} E_{\alpha}^{ \pm} f$ satisfying, for $\varepsilon \equiv \varepsilon(N)$ small enough,

$$
\left\|E_{\alpha}^{ \pm} f-f_{N, \varepsilon}^{ \pm}\right\|_{L^{2}\left([0,1]^{2}\right)}^{2} \leq C N^{-\alpha},
$$

for some constant $C>0$ independent of $N$ and on Delaunay triangulations $\mathcal{D}_{N, \varepsilon}$ which are a subdivision of the perturbed Birman-Solomjak quadtree partitions $\mathcal{Q}_{N, \varepsilon}$.

This finally leads us to the stated estimates

$$
\left\|E_{\alpha}^{ \pm} f-f_{N, \varepsilon}^{ \pm}\right\|_{L^{2}\left(\Omega_{N}^{ \pm}\right)}^{2}=\left\|f-f_{N, \varepsilon}\right\|_{L^{2}\left(\Omega_{N}^{ \pm}\right)}^{2} \leq C N^{-\alpha},
$$

which, in combination with (5.5) and (5.6), completes our proof by $\mathcal{D}_{N}:=\mathcal{D}_{N, \varepsilon}$ and $f_{N}:=f_{N, \varepsilon}$.

Note that the set $\mathscr{H}^{\alpha, 2}\left([0,1]^{2}\right)$ of generalized $\alpha$-horizon functions (cf. Definition 5.1) contains the set of $\alpha$-horizon functions (cf. Definition 3.1). Therefore, the error bound in (5.4) is optimal in exactly the same sense as discussed at the end of Section 3: no depth-search limited dictionary can achieve a better asymptotic decay rate than that in (5.4).

\section{ACKNOWLEDGEMENT}

The second author is partly supported by the priority program DFG-SPP 1324 of the Deutsche Forschungsgemeinschaft (DFG) within the project IS 58/1-2. We thank Felix Henneke for his useful assistance during an early stage of our research.

\section{REFERENCES}

1. R. Adams and J. Fournier: Sobolev Spaces. Academic Press, 2003.

2. F. Arandiga, A. Cohen, R. Donat, N. Dyn, and B. Matei: Approximation of piecewise smooth functions and images by edge-adapted (ENO-EA) nonlinear multiresolution techniques. Appl. Comput. Harmon. Anal. 24, 2008, 225-250.

3. C. Benett and R. Sharpley: Interpolation of Operators. Academic Press, 1998.

4. M. Birman and M. Solomjak: Piecewise-polynomial approximations of functions of the classes $W_{p}^{\alpha}$. Math. USSR-Sbornik 2(3), 1967.

5. S. Bougleux, G. Peyré, and L. Cohen: Image compression with anisotropic geodesic triangulations. Proceedings of ICCV'09, Oct. 2009.

6. E.J. Candès, L. Demanet, D.L. Donoho, and L. Ying: Fast discrete curvelet transforms. Multiscale Model. Simul. 5, 2006, 861-899.

7. E. Candès, and D. Donoho: New tight frames of curvelets and optimal representations of objects with piecewise $C^{2}$ singularities. Comm. Pure Appl. Math. 57(2), 2004, 219-266.

8. A. Cohen, N. Dyn, F. Hecht, and J.-M. Mirebeau: Adaptive multiresolution analysis based on anisotropic triangulations. Math. Comp. 81, 2012, 789-810 
9. A. Cohen and J.-M. Mirebeau: Greedy bisection generates optimally adapted triangulations. To appear in Mathematics of Computation.

10. S. Dekel and D. Leviatan: Adaptive multivariate approximation using binary space partitions and geometric wavelets. SIAM J. Numer. Anal. 43, 2006, 707-732.

11. L. Demaret, N. Dyn, and A. Iske: Image compression by linear splines over adaptive triangulations. Signal Processing Journal 86(7), July 2006, 1604-1616.

12. L. Demaret and A. Iske: Anisotropic triangulation methods in adaptive image approximation. In: Approximation Algorithms for Complex Systems, E.H. Georgoulis, A. Iske, and J. Levesley (eds.), Springer, Berlin, 2011, 47-68.

13. L. Demaret and A. Iske: Adaptive image approximation by linear splines over locally optimal Delaunay triangulations. IEEE Signal Processing Letters 13(5), May 2006, 281-284.

14. R. DeVore: Nonlinear approximation. Acta Numerica 7, 1998, 51-150.

15. R. DeVore, B. Jawerth, and B. Lucier: Image compression through wavelet transform coding. IEEE Transactions on Information Theory 38(2), 1992.

16. M.N. Do and M. Vetterli: The contourlet transform: an efficient directional multiresolution image representation. IEEE Transactions on Image Processing 14(12), December 2005, 20912106.

17. D. Donoho: Wedgelets: nearly-minimax estimation of edges. Ann. Stat. 27, 1999, 859-897.

18. D. Donoho: Sparse components of images and optimal atomic decompositions, Constructive Approximation, vol, 17, 2001,353-382.

19. H. Edelsbrunner and E. Mücke: Simulation of simplicity: a technique to cope with degenerate cases in geometric algorithms. ACM Transactions on Graphics 9(1), 1990, 66-104.

20. D.E. Edmunds and H. Triebel: Function Spaces, Entropy Numbers and Differential Operators. Cambridge University Press, Cambridge, UK, 1996.

21. K. Guo and D. Labate: Optimally sparse multidimensional representation using shearlets. SIAM J. Math. Anal. 39, 2007, 298-318.

22. K. Guo, W.-Q. Lim, D. Labate, G. Weiss, and E. Wilson: Wavelets with composite dilations. Electr. Res. Announc. of AMS 10, 2004, 78-87.

23. L. Jaques and J.-P. Antoine: Multiselective pyramidal decomposition of images: wavelets with adaptive angular selectivity. Int. J. Wavelets Multiresolut. Inf. Process. 5, 2007, 785-814.

24. A. Korostelev and A. Tsybakov: Minimax Theory of Image Reconstruction, Springer, 1993.

25. B. Lehner, G. Umlauf, and B. Hamann: Image compression using data-dependent triangulations. In: Advances in Visual Computing, G. Bebis et al. (eds.), Springer, LNCS 4841, 2007, 351-362.

26. E. Le Pennec and S. Mallat: Bandelet image approximation and compression. Multiscale Model. Simul. 4, 2005, 992-1039.

27. S. Mallat: A Wavelet Tour of Signal Processing. Academic Press, 2nd Edition, 1999.

28. S. Mallat: Geometrical grouplets. Appl. Comput. Harmon. Anal. 26, 2009, 161-180.

29. G. Plonka: The easy path wavelet transform: a new adaptive wavelet transform for sparse representation of two-dimensional data. Multiscale Modelling Simul. 7, 2009, 1474-1496.

30. D.D. Po and M.N. Do: Directional multiscale modeling of images using the contourlet transform. IEEE Trans. Image Process. 15, 2006, 1610-1620.

31. F.P. Preparata and M.I. Shamos: Computational Geometry. Springer, New York, 1988.

32. R. Shukla, P.L. Dragotti, M.N. Do, and M. Vetterli: Rate-distortion optimized tree structured compression algorithms for piecewise smooth images. IEEE Trans. Image Process. 14, 2005, 343-359.

33. M.B. Wakin, J.K. Romberg, H. Choi, and R.G. Baraniuk: Wavelet-domain approximation and compression of piecewise smooth images. IEEE Trans. Image Process. 15, 2006, 1071-108. 